\title{
Osteopontin Augments M2 Microglia Response and Separates M1- and M2-Polarized Microglial Activation in Permanent Focal Cerebral Ischemia
}

\author{
Anne Ladwig, ${ }^{1}$ Helene Luise Walter, ${ }^{1}$ Jörg Hucklenbroich, ${ }^{1}$ Antje Willuweit, ${ }^{2}$ \\ Karl-Josef Langen, ${ }^{2}$ Gereon Rudolph Fink, ${ }^{1,3}$ Maria Adele Rueger, ${ }^{1,3}$ and \\ Michael Schroeter ${ }^{1,3}$ \\ ${ }^{1}$ Department of Neurology, University Hospital of Cologne, Cologne, Germany \\ ${ }^{2}$ Medical Imaging Physics, Institute of Neuroscience and Medicine (INM-4), Research Centre Juelich, Juelich, Germany \\ ${ }^{3}$ Cognitive Neuroscience, Institute of Neuroscience and Medicine (INM-3), Research Centre Juelich, Juelich, Germany \\ Correspondence should be addressed to Michael Schroeter; michael.schroeter@uk-koeln.de
}

Received 7 June 2017; Revised 30 July 2017; Accepted 29 August 2017; Published 20 September 2017

Academic Editor: Michal A. Rahat

Copyright (C) 2017 Anne Ladwig et al. This is an open access article distributed under the Creative Commons Attribution License, which permits unrestricted use, distribution, and reproduction in any medium, provided the original work is properly cited.

Background. Focal cerebral ischemia induces distinct neuroinflammatory processes. We recently reported the extracellular phosphor-glyco-protein osteopontin (OPN) to directly affect primary microglia in vitro, promoting survival while shifting their inflammatory profile towards a more neutral phenotype. We here assessed the effects of OPN on microglia after stroke in vivo, with focus on infarct demarcation. Methods. Animals underwent focal photothrombotic stroke and were injected intracerebroventricularly with $500 \mu \mathrm{g}$ OPN or vehicle. Immunohistochemistry assessed neuronal damage and infarct volume, neovascularisation, glial scar formation, microglial activation, and M1 and M2 polarisation. Results. After photothrombotic stroke, areas covered by M1 and M2 microglia substantially overlapped. OPN treatment reduced that overlap, with microglia appearing more spread out and additionally covering the infarct core. OPN additionally modulated the quantity of microglia subpopulations, reducing iNOS+ M1 cells while increasing M2 microglia, shifting the M1/M2 balance towards an M2 phenotype. Moreover, OPN polarized astrocytes towards the infarct. Conclusion. Microglial activation and M1 and M2 polarization have distinct but overlapping spatial patterns in permanent focal ischemia. Data suggest that OPN is involved in separating M1 and M2 subpopulations, as well as in shifting microglia polarization towards the M2 phenotype modulating beneficially inflammatory responses after focal infarction.

\section{Introduction}

Focal cerebral ischemia induces an activation of brain resident glial cells and an influx of peripheral immune cells [1]. Those inflammatory responses have been termed a "doubleedged sword" since they not only serve beneficial functions, such as phagocytosis of debris and recruitment of neural stem cells, but also mediate secondary tissue damage [2]. Multiple activational states of microglia are often coined in a dichotomous way as M1 and M2 subtypes, with more proinflammatory and detrimental effects attributed to the M1 phenotype while more regulatory and protective actions attributed to M2 phenotypes [3]. We previously characterized the process by which microglia as the resident immune cells of the brain separate vital brain tissue from ischemic necrosis [4]. At the very margin of those two, the "demarcation zone" is characterized by microglia actively secreting the nerve/glial antigen 2 (NG2) into the extracellular matrix (ECM), thereby separating necrosis from vital tissue with a decisive impact on secondary tissue damage [4]. Recently, Gliem et al. reported the ECM glycoprotein osteopontin (OPN) to be involved in the demarcation of focal ischemic lesions by elongating astrocytic processes, improving astrocyte-mediated neovessel coverage [5]. Our own recent 
TABLE 1: Details of primary antibodies.

\begin{tabular}{|c|c|c|c|c|}
\hline Antibody & Antigene & Dilution/fixation & Identified cell type & Reference, company \\
\hline Anti-NeuN & $\begin{array}{l}\text { Neuronal nuclear antigen } \\
\text { (DNA-binding) }\end{array}$ & $\begin{array}{c}1: 20004 \% \text { PFA, } \\
(2 \mathrm{~h})\end{array}$ & Surviving neurons & $\begin{array}{l}\text { Anti-NeuN monoclonal antibody, mouse, } \\
\text { catalog number MAB377, Merck Millipore }\end{array}$ \\
\hline Anti-Ibal & $\begin{array}{l}\text { Ionized calcium binding } \\
\text { adaptor molecule } 1\end{array}$ & $\begin{array}{l}1: 1000,4 \% \text { PFA, } \\
(2 \mathrm{~h})\end{array}$ & $\begin{array}{l}\text { Activated microglia/ } \\
\text { macrophages }\end{array}$ & $\begin{array}{c}\text { Anti-ibal polyclonal antibody, rabbit, catalog } \\
\text { number 019-19741, Wako Chemicals }\end{array}$ \\
\hline Anti-iNOS & Inducible NO-synthetase & $\begin{array}{l}1: 100,4 \% \text { PFA, } \\
\quad(15 \mathrm{~min})\end{array}$ & $\begin{array}{l}\text { M1-polarized } \\
\text { microglia }\end{array}$ & $\begin{array}{l}\text { Anti-iNOS polyclonal antibody, rabbit, } \\
\text { catalog number ab15323, abcam }\end{array}$ \\
\hline Anti-Arg1 & Arginase 1 & $\begin{array}{l}1: 10004 \% \text { PFA, } \\
\quad(15 \mathrm{~min})\end{array}$ & $\begin{array}{l}\text { M2-polarized } \\
\text { microglia }\end{array}$ & $\begin{array}{l}\text { Anti-liver-arginase polyclonal antibody, } \\
\text { goat, catalog number ab60176, abcam }\end{array}$ \\
\hline Anti-CD206 & Mannose receptor & $\begin{array}{l}1: 1500,4 \% \text { PFA, } \\
\quad(30 \mathrm{~min})\end{array}$ & $\begin{array}{l}\text { M2-polarized } \\
\text { microglia }\end{array}$ & $\begin{array}{l}\text { Anti-CD206 polyclonal antibody, goat, } \\
\text { catalog number AF2535, R\&D Systems }\end{array}$ \\
\hline Anti-Ym1 & Chitinase-3-like-3 & $\begin{array}{l}1: 100,50 \% \text { acetone } \\
\left(15 \mathrm{~min}, 4^{\circ} \mathrm{C}\right) 0.3 \% \mathrm{TX}\end{array}$ & $\begin{array}{l}\text { M2-polarized } \\
\text { microglia }\end{array}$ & $\begin{array}{c}\text { Anti-Ym1 polyclonal, rabbit, catalog number } \\
01404 \text {, Stemcell Technologies }\end{array}$ \\
\hline Anti-vWF & von Willebrand factor & $\begin{array}{l}1: 1500,4 \% \text { PFA, } \\
\quad(15 \mathrm{~min})\end{array}$ & Endothelial cells & $\begin{array}{l}\text { Anti-vWF polyclonal antibody, rabbit, } \\
\text { catalog number ab6994, abcam }\end{array}$ \\
\hline Anti-NG2 & $\begin{array}{l}\text { Chondroitin sulfate } \\
\text { proteoglycan }\end{array}$ & $\begin{array}{l}1: 4000,100 \% \text { acetone } \\
\quad\left(10 \mathrm{~min},-20^{\circ} \mathrm{C}\right)\end{array}$ & Polydendrocytes & $\begin{array}{l}\text { Anti-NG2 polyclonal antibody, rabbit, } \\
\text { catalog number AB5320, Millipore }\end{array}$ \\
\hline Anti-GFAP & $\begin{array}{l}\text { Glial fibrillary acidic } \\
\text { protein }\end{array}$ & $\begin{array}{l}1: 2000,4 \% \mathrm{PFA} \\
\text { (overnight, } 4^{\circ} \mathrm{C} \text { ) }\end{array}$ & Astrocytes & $\begin{array}{l}\text { Anti-GFAP monoclonal antibody, mouse, } \\
\text { catalog number MAB360, Millipore }\end{array}$ \\
\hline
\end{tabular}

work revealed direct effects of OPN on primary microglia in vitro, promoting their survival while shifting their inflammatory profile towards a more neutral phenotype [6]. Although exact protein concentrations are unknown, OPN protein is virtually absent in the naïve brain, but OPN mRNA is upregulated by some 5000 times in the infarct area after focal cerebral ischemia. The absence of OPN has been shown to increase secondary neurodegeneration [7]. Based on these observations, we hypothesized that OPN would modulate the inflammatory profile of microglia after stroke in vivo, while potentially affecting the process of infarct demarcation as well.

\section{Methods}

\subsection{Animals and Surgery}

2.1.1. Focal Ischemia Model: Photothrombosis (PT). Adult male Fisher 344 rats $(n=15)$ weighing $200-300 \mathrm{~g}$ were sedated with isoflurane (2-5\%), subsequently anesthetized with i.p. ketamine $(75 \mathrm{mg} / \mathrm{kg}) /$ medetomidine $(0.5 \mathrm{mg} / \mathrm{kg})$ and atraumatically fixated in a stereotactic frame.

After disinfection, the scalp was longitudinally incised $(10 \mathrm{~mm})$ and the skull was exposed. Periost was removed leaving the bone intact. Bregma and lambda points were identified. A fiberoptic bundle of a cold light source with an aperture of $1.5 \mathrm{~mm}$ was placed onto the skull using a micromanipulator and adjusted to the stereotactic coordinates $2 \mathrm{~mm}$ posterior and $3 \mathrm{~mm}$ lateral from the bregma. The skull was illuminated for 20 minutes with a white light beam $(150 \mathrm{~W})$. During the first 2 minutes of illumination, the rats were injected with the dye rose bengal (Sigma, Munich, Germany $1 \%, 1 \mu \mathrm{g}$ per g bodyweight).

Following surgery, the wound was sutured and the animals were allowed to recover from anaesthesia. Afterwards, they were put back into their home cages and were given access to food and water ad libitum.

2.1.2. OPN Treatment. To assess the effects of OPN in cerebral ischemia in vivo, one day after induction of photothrombosis, the animals were randomly assigned to the treatment groups and treated with a single intracerebroventricular (i.c.v.) injection of either $500 \mu \mathrm{g}$ recombinant OPN (R\&D Systems) in $5 \mu \mathrm{l}$ saline $(n=8)$, or $5 \mu \mathrm{l}$ saline as control (placebo group, $n=7$ ). To this end, each animal was put under anaesthesia with ketamine $(75 \mathrm{mg} / \mathrm{kg})$ and medetomidine $(0.5 \mathrm{mg} / \mathrm{kg})$ and injected stereotactically using the following coordinates: bregma anteroposterior $-0.9 \mathrm{~mm}$, mediolateral $+1.4 \mathrm{~mm}$, and ventrodorsal $+3.8 \mathrm{~mm}$.

After injection, the animals were allowed to recover from anaesthesia and were returned to their cages, where they were given access to food and water ad libitum.

Eight days post i.c.v. injection, rats were sacrificed under deep anaesthesia.

2.2. Histology and Immunohistochemistry. Serial coronal brain sections (slice thickness $20 \mu \mathrm{m}$ ) were cut through the area of greatest infarct diameter on a cryostat (CM3050S, Leica Instruments).

To assess the patterns of inflammatory cell infiltration in MCAO and photothrombosis, brain sections were stained with a selection of antibodies against an M1- and M2-polarized microglia (Table 1). Microglia stainings were supplemented by stainings against antineuronal nuclear $(\mathrm{NeuN})$ antigen to assess the extent of ischemic damage, von Willebrand factor (vWF) for the identification of (neo-)vascularisation, the astrocytic marker GFAP, and anti-NG2, an antigen recognized on oligodendrocyte and microglial precursors (Table 1).

Depending on the antibody, the sections were either fixed with $4 \%$ PFA for $15 \mathrm{~min}, 30 \mathrm{~min}$ at room temperature (RT), 
or overnight at $4^{\circ} \mathrm{C}$. All following incubation steps were conducted at RT unless mentioned otherwise. After fixation, the slides were washed in $0.1 \mathrm{M}$ PBS, quenched in $\mathrm{H}_{2} \mathrm{O}_{2}$ (only in $3,3^{\prime}$-diaminobenzidine (DAB) staining), washed in $0.1 \mathrm{M}$ PBS again, and blocked with 5\% normal goat serum, 5\% normal donkey serum (Jackson Immuno Research Laboratories, Baltimore, USA), or 5\% normal horse serum (Vector Laboratories, Burlingame, CA, USA) in 0.1 M PBS (with or without $0.3 \%$ Triton $\mathrm{X}$ ) for $45 \mathrm{~min}$. The sections were incubated with the primary antibody overnight at $4^{\circ} \mathrm{C}$ (dilutions are detailed in Table 1).

After rinsing and washing in $0.1 \mathrm{M} \mathrm{PBS}$, the secondary antibody was applied and incubated for $30 \mathrm{~min}$. Secondary antibodies used were biotinylated goat anti-rabbit IgG, biotinylated horse anti-mouse IgG, and biotinylated horse anti-goat IgG (Vector Laboratories, Burlingame, CA, USA).

For light microscopic visualization, the ABC Elite Kit (Vector Laboratories) followed by diaminobenzidine (Sigma Aldrich, Munich, Germany) as final reaction products were used.

Secondary antibodies for fluorescence microscopy were donkey anti-rabbit 488 IgG, donkey anti-goat 568 IgG, donkey-anti-mouse 488 IgG, goat-anti-mouse 488, and goat-anti rabbit 568 (Alexa Fluor, Invitrogen/Life Technologies, Carlsbad, CA, USA). For nuclear costaining, Hoechst 33342 (Life Technologies, Darmstadt, Germany) was applied for $10 \mathrm{~min}$ after rinsing the secondary antibody.

Representative images were taken using an inverted fluorescence phase-contrast microscope (Keyence BZ-9000E, Osaka, Japan) with 4x, 20x, and 40x objectives.

2.3. Image Analysis. Sequentially scanned images of the infarct area were reconstructed in the BZ-II Analyzer (Keyence, Neu-Isenburg, Germany). All measurements and evaluations were performed by a blinded evaluator (A.L.) with the open-source software ImageJ/Fiji (Rasband, W.S., ImageJ, US National Institutes of Health, Bethesda, Maryland, USA, https://imagej.nih.gov/ij/, 1997-2014). The interrater reproducibility of the threshold measurements was verified by remeasurements of randomly chosen samples by a second investigator (H.L.W.).

2.3.1. Area Measurements. In order to evaluate the percentage of the infarct area covered by a certain cell type, the infarct area (delineated by NeuN staining) was marked with the polygon selections tool and cut out. Subsequently, the threshold tool was used to selectively mark the cell-like formations stained with the respective antibody and to subtract the background. The total infarct area and the remaining area were measured in $\mu \mathrm{m}^{2}$, and the percentage was calculated using Microsoft Excel 2011.

2.3.2. Cell Counts and Area Measurements in Specific Infarct Zones. To quantify the observed changes in stratification of the inflammatory cells within the infarct area (Figure 1), cell densities and areas covered by one cell type were measured within the respective infarct zone. Zones were defined according to the Ibal staining. The densest Ibal belt defined the infarct margin at days 8-10 after ischemia as described earlier [4]. The area between the hypocellular core and the infarct margin was labelled as the outer infarct core. The area delineating necrotic tissue from viable tissue as defined by NeuN-positive neurons was termed as the demarcation zone [4]. Outside of the infarct border laid the peri-infarct zone, which was characterized by a continuously increasing density of Iba1+ cells toward the infarct. It transitioned into the unaffected tissue. Representative fields of view (measuring $100 \times 100 \mu \mathrm{m}$ ) were chosen from each animal from each zone, and the area covered by the individual cell type within this $10000 \mu \mathrm{m}^{2}$ square was measured.

2.3.3. Planimetry. To assess the effect of OPN on absolute and relative size of each infarct zone, the Iba1-stained slides were used, and measurements were performed using the polygonal section tool from ImageJ/Fiji.

2.3.4. Generation of Pseudocolored Overlays. Adjacent images stained with all mentioned antibodies were edited with the threshold tool of ImageJ/Fiji. In a picture-editing program (Pixelmator 3.3 for Mac), those images were assigned pseudocolors and adjacent sections were aligned manually with the aid of anatomical landmarks.

2.3.5. GFAP Polarization and Measurement. GFAP-positive cells, which were preserved and stained completely, were counted and assigned to a polarized or unpolarized group depending on their process alignment [5]. The processes were measured.

2.4. Statistical Analysis. Statistical evaluations were performed using the GraphPad Prism software. Data sets were tested for normal distribution with the D'Agostino-Pearson normality test. For normally distributed data sets, a parametric test was used (unpaired $t$-test for two samples). For unequal variances between two samples, Welch's $t$-test was assigned. A nonparametric test was used for nonnormally distributed data sets (Mann-Whitney test for two samples). $p$ value was set at 0.05 for statistical significance.

\section{Results}

3.1. Functional Layering of Microglia Subtypes. Neuroinflammation helped to define multiple subareas of the infarct tissue organized in centrifugal order: hypocellular infarct core, outer infarct core, infarct margin, demarcation zone, and peri-infarct zone (Figure 2(a)). The closer to the infarct margin, the more microglia were activated. The distribution of differently polarized subtypes of microglia-M1 and $\mathrm{M} 2$ - in and around the ischemic lesion was assessed immunohistochemically. Pseudocolors were assigned to each marker to visualize the distribution of M1- and M2polarized microglia in OPN-treated and control brains (Figure 2(c)). In controls, neuroinflammation was confined to a comparatively thin belt around the necrotic infarct core, with the different microglial subpopulations overlaying each other to a great extent (Figure 2(c)). In OPN-treated animals, microglial subpopulations overlapped less and rather spread out into the lesion itself (Figure 2(c)). In particular, Arg1+ M2 microglia extended centripetally into the infarct 

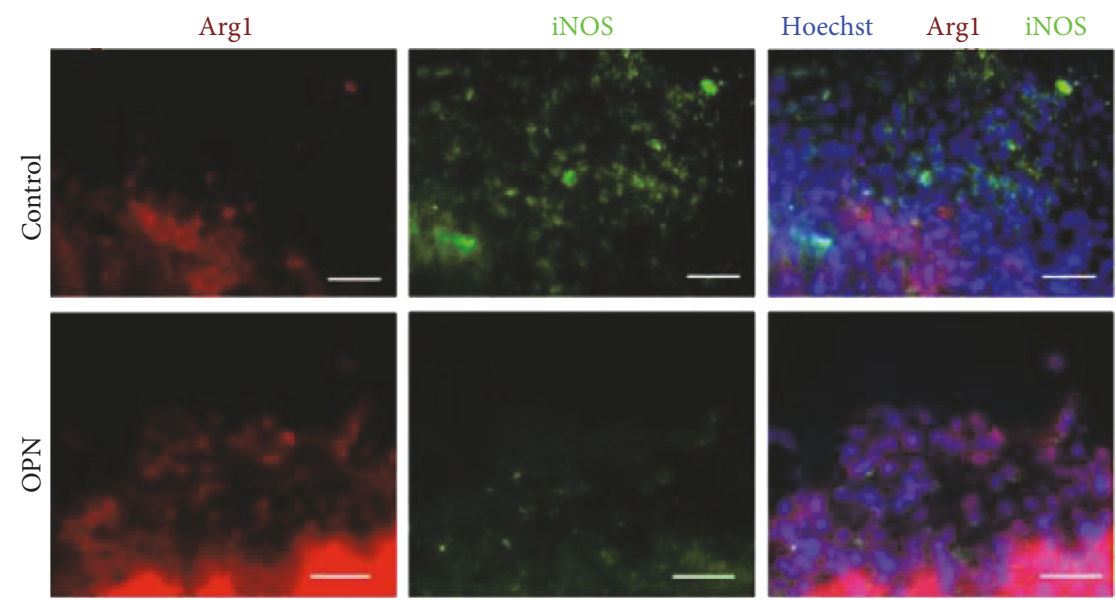

(a)

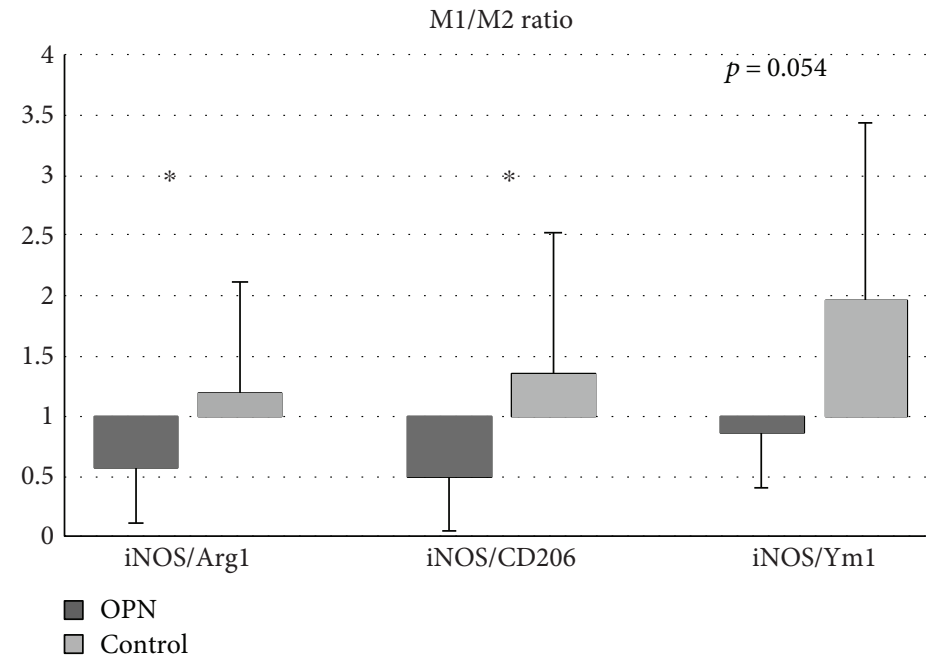

(b)
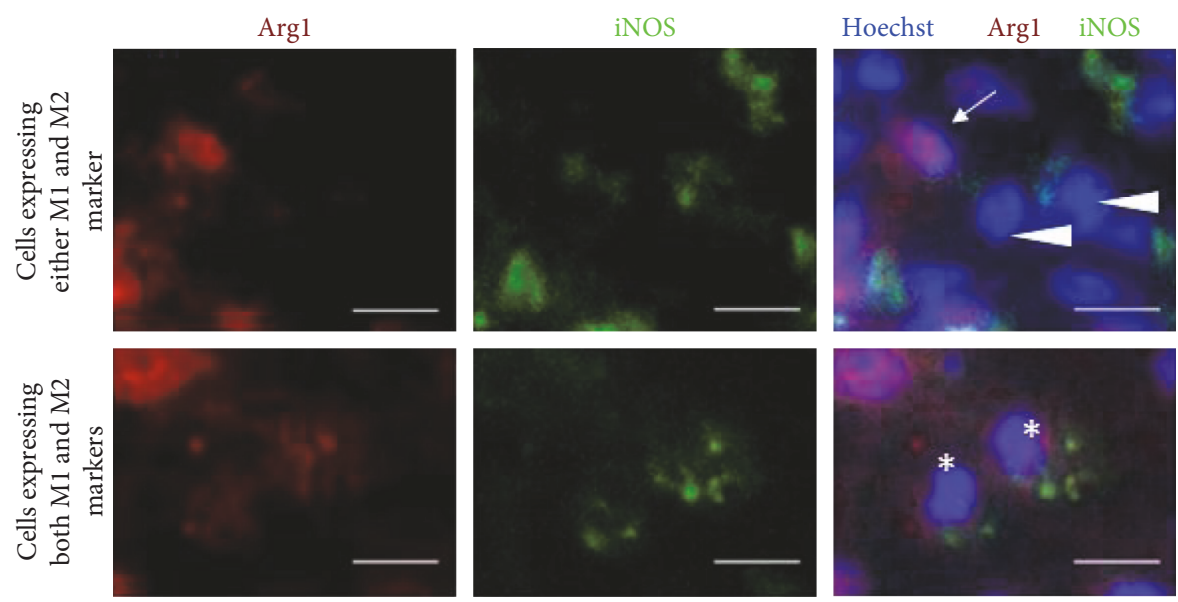

(c)

FIGURE 1: OPN treatment shifts the M1/M2 balance towards an M2 phenotype. (a) Arg1+ M2 cells (red) dominated the infarcted area in OPN-treated rats, whereas iNOS+ M1 microglia were found predominantly in vehicle-treated animals (green). (b) M1 (iNOS-expressing) microglia and M2 (Arg+, CD206+, or Ym1+) microglia in the infarcted area after photothrombosis expressed as M1/M2 ratio. Ten days after photothrombosis, the ratios of M1/M2 microglia were significantly decreased in OPN-treated animals compared to vehicle-treated controls, suggesting an increase in M2 polarization. (c) Close-up of microglia expressing either M1 (iNOS) or M2 (Arg1) markers (upper row), or expressing both markers (lower row). Scale bars represent $40 \mu \mathrm{m}$ in (a) and $10 \mu \mathrm{m}$ (c). All graphs show mean and SD; ${ }^{*} p<0.05$. 


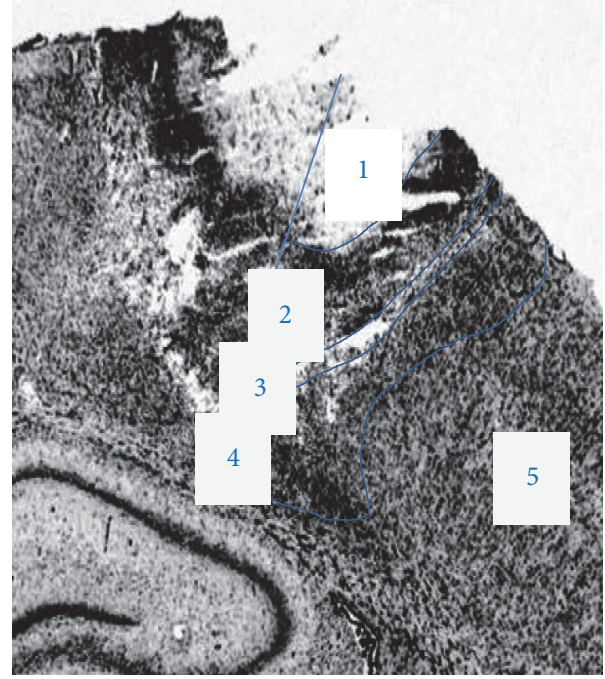

(a)
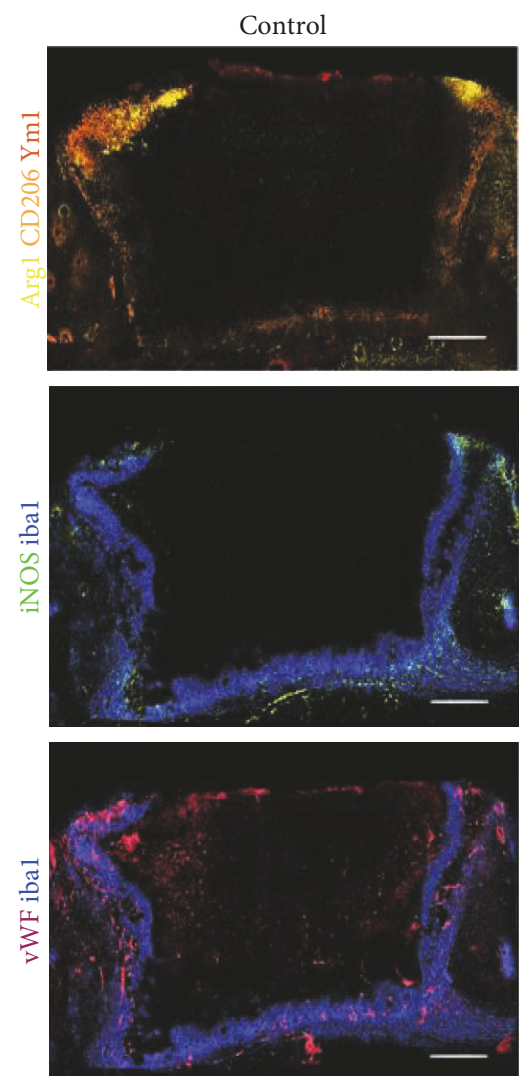
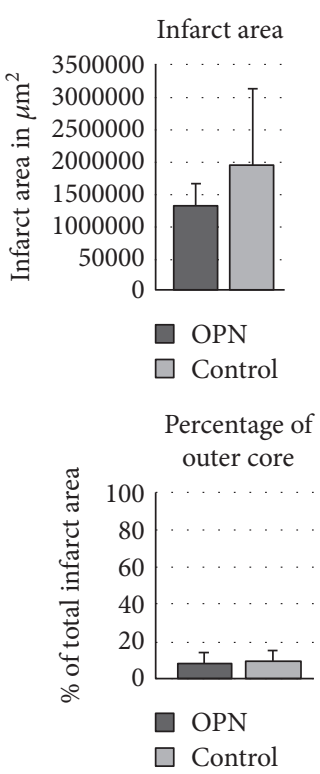

(b)
Percentage of

hypocellular core

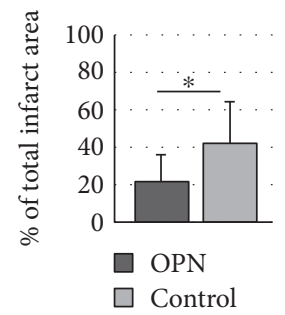

Percentage of

infarct margin

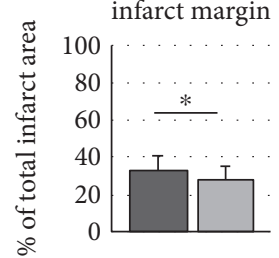

$\square$ OPN

$\square$ Control

)
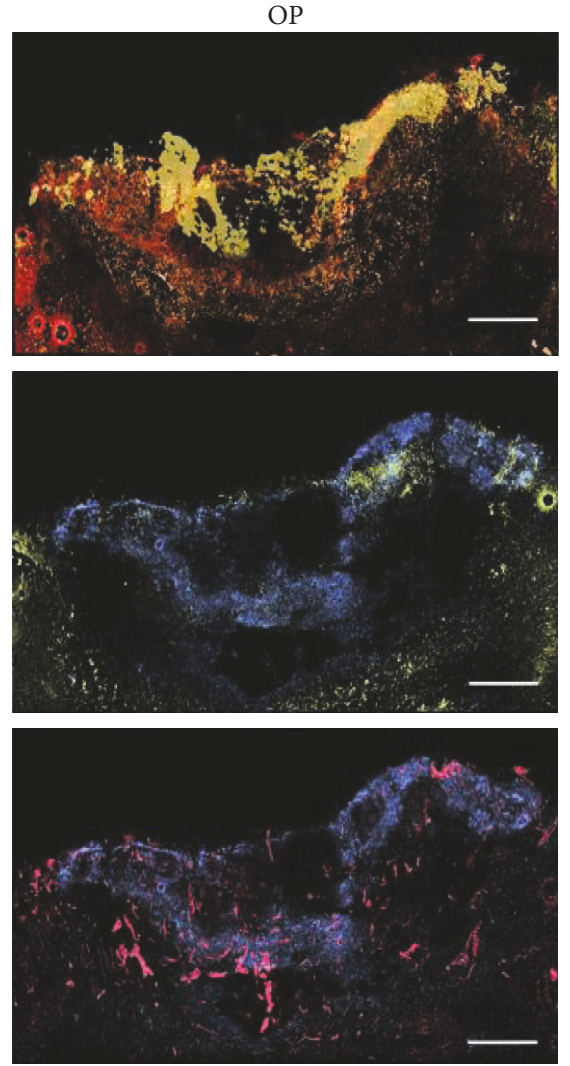

(c)

Figure 2: OPN increases functional layering of microglia subtypes and influences the relative size of distinct infarct subareas. (a) Cresyl violet staining of the photothrombotic infarct. The infarct area could be subdivided into five zones according to distribution pattern of inflammatory cells: hypocellular infarct core (1), cell-rich infarct margin (2), demarcation zone (3), peri-infarct zone (4), and unaffected tissue (5). (b) The size of the evaluated infarct area shows a trend towards smaller infarcts, but no significant changes under OPN treatment. The infarct layers, especially those close to the core show significant changes. The size of the hypocellular core is significantly reduced post OPN and the infarct margin is proportionally larger. (c) Displays pseudocoloured images of exemplary photothrombotic infarcts. Sections were stained for Arg1 (yellow), CD206 (orange), YM1 (red), iNOS (green), Iba1 (blue), and vWF (purple). Adjacent sections of an OPN-treated animal (right column) and a control rat (left column). Scale bars represent $300 \mu \mathrm{m}$ (c). All graphs show mean and SD; ${ }^{*} p<0.05$. 
core, diminishing the hypocellular part to a minimum (Figure 2(c)). Thus, OPN induced a more pronounced stratification of inflammatory cells within the infarct area.

3.2. Relative Sizes of Distinct Infarct Subareas. Immunohistochemical data were used to quantify the relative size of the distinct infarct subareas in control versus OPNtreated animals. After OPN injection, we observed a smaller hypocellular infarct core $(p=0.0045)$ but a wider infarct margin $(p=0.0407)$ than under control conditions (Figure 2(b)).

3.3. Infiltration of Microglia Subpopulations into Distinct Infarct Subareas. The ischemic lesion was subdivided into five distinct regions (Figure 2(a)). In the outer infarct core, general Iba1+ activated microglia were more pronounced after OPN-treatment than under control conditions $(p=$ 0.0008 , Figure 3(a)), and the same was observed for M2polarized microglia (Figure 4), suggesting OPN to activate microglia and induce their M2 polarization. In parallel, and particularly in the infarct margin, expression of iNOS by M1 microglia was decreased by OPN (Figure 3(b)).

3.4. Ratio between M1 and M2 Microglia. Ratios between M1and M2-polarized microglia were assessed on the basis of immunohistochemical stainings for the M1 marker iNOS and the M2 markers Arg1, CD206, and Ym1. Arg1+ M2 cells dominated the infarct in OPN-treated rats, whereas iNOS+ M1 microglia were found predominantly in control animals (Figure 1(a)). For all three combinations (iNOS/ Arg1, $p=0.0406$, iNOS/CD206, $p=0.0401$, and iNOS/ Ym1, $p=0.054), \mathrm{M} 1 / \mathrm{M} 2$ ratios were decreased following i.c.v. injection of OPN into stroke rats (Figure 1(b)). We observed not only cells expressing either M1 or M2 markers but also others expressing both markers at once (Figure 1(c)).

3.5. Astrocyte Polarization. GFAP+ astrocytes forming the glial scar around the infarct adopted a bipolar form in OPN-treated animals (Figures 5(a) and 5(b)). Polarized astrocytes were characterized by an elongated shape, pointing their processes towards the infarct core. The length of the cellular processes was significantly increased after OPN compared to controls (Figure 5(c)).

\section{Discussion}

Permanent focal cerebral ischemia elicits a profound neuroinflammatory reaction dominated by resident microglia and macrophages recruited from the blood stream. This reaction is initiated immediately after the onset of ischemia and lasts for weeks [8]. Inflammation occurring in defined spatiotemporal patterns strongly impacts on the outcome after ischemia [9-11].

4.1. Microglial Activation. The microglia/macrophage population is heterogeneous not only in origin but also in morphology and function. Upon activation, microglia undergo characteristic morphological changes. In a sequence of events, they shift from a resting ramified phenotype via a transient hyperramified phenotype towards a stellate and later ameboid shape. Microglial activation reflects the severity of tissue damage and may well extend beyond the site of primary tissue damage. The description of previous studies had already described two nonoverlapping subpopulations of CD4+ and CD8+ microglia/macrophages in rats, providing early evidence for heterogeneous microglia/macrophage populations in poststroke inflammation [12-15]. As described more recently, activated microglia may functionally polarize towards an $\mathrm{M} 1+$ or M2+ cell phenotype $[3,16,17$. The direction of polarization is essentially influenced by the surrounding "milieu" $[18,19]$. In an inflammatory setting, OPN might be a suitable candidate for establishing such a "milieu" [20]. Being secreted into the ECM by microglia, it acts through paracrine and autocrine signalling, thereby activating microglia and interacting with other cells that impact on regeneration, such as astrocytes, neural stem cells, and endothelial cells [20-22].

4.2. M1 And M2 Polarization. We here describe that polarization of microglia towards an M1 and or M2 phenotype could be recognized in photothrombosis, extending earlier results in focal ischemia [23]. In the photothrombotic infarct, we detected M1 microglia predominantly in the peri-infarct area at in the vicinity of the demarcation zone. M1 microglia is thought to produce oxygen species and generally thus create a toxic environment [24]. The M1 shift goes along with a switch in mitochondrial energy production from oxidative phosphorylation to anaerobic glycolysis further increasing metabolic demands, lactate acidosis, and free radical production [3]. The localization of M1 microglia with high energy demand in the peri-infarct zone reported here-and given functional connotations of M1 microglia-make these cells likely to contribute to secondary damage after focal ischemia.

Ineffective phagocytic activity of microglia goes along with the failure of containment of necrotic tissue, causing ongoing microglial activation and loss of trophic support for surrounding neurons; these processes can cause further bystander damage, as is known from neurodegenerative diseases [25-27]. M2 polarization is suggested to be the more "benign" activation state, accelerating removal of debris and repairing damaged tissue. We here observed M2 microglia within the necrotic infarct core area and in close proximity to sites of neovascularisation.

Of note, in our study, microglial activation did not necessarily go along with either M1 or M2 polarization. An essential proportion of Iba1+, microglia exhibited stellate or ameboid morphology but expressed neither M1nor M2-typical markers. This is in accordance with the hypothesis of multistep und tightly regulated microglial activation resulting in multiple functional and metabolic subtypes [28].

4.3. OPN Effects. We found a decreased ratio of M1 and M2, implying a shift towards M2 microglia cells 10 days after the induction of ischemia. We here investigated whether the M1 to M2 shift was a fluent transition, or represented two distinct nonoverlapping populations that 
Ibal (activated)
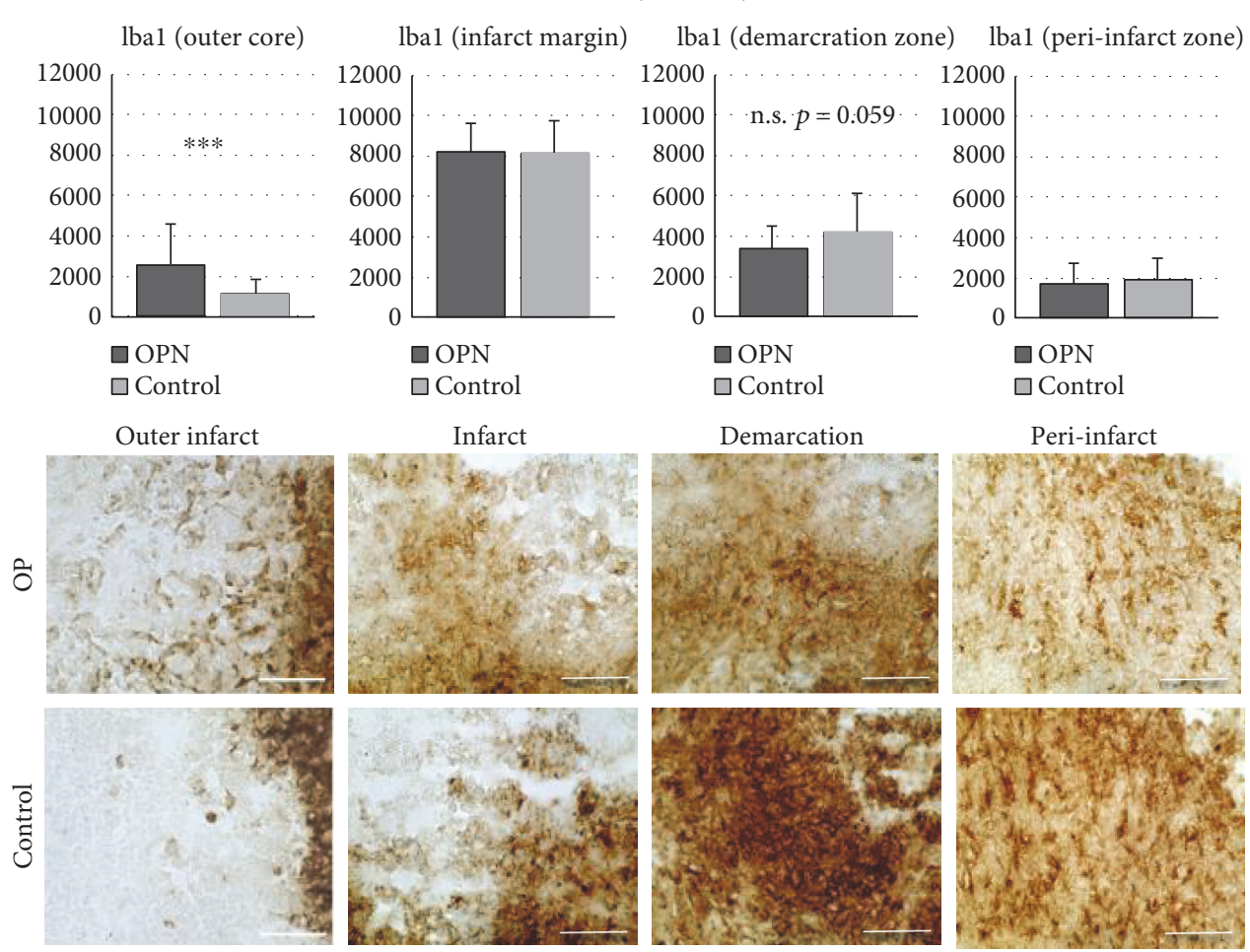

(a)

iNOS (M1-polarized)

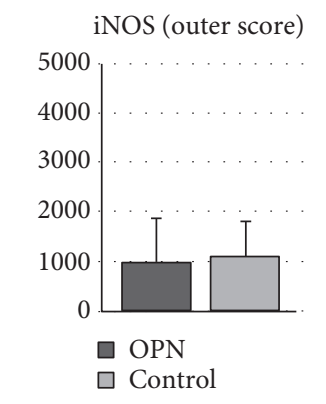

iNOS (infarct margin)
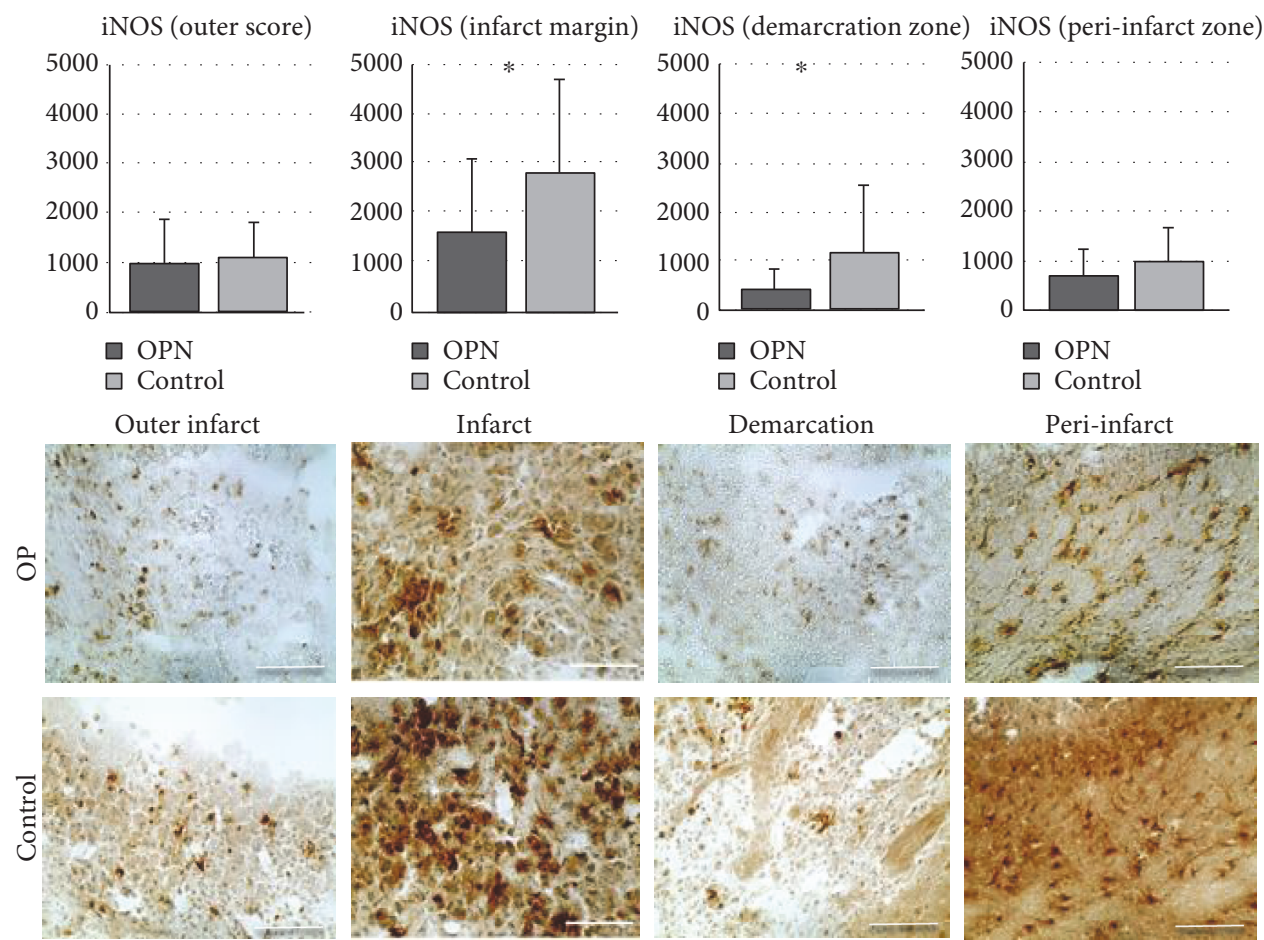

(b)

FIGURE 3: OPN modulates the infiltration of microglia subpopulations into distinct infarct subareas (part I: Iba1 and iNOS). All microglia stained with Iba1+ (a) and proinflammatory microglia stained with iNOS (b) shown in distinct subareas of the photothrombotic infarct after OPN treatment compared to control. Iba1+ cells are found elevated the outer infarct core, while iNOS+ cells (M1) are decreased in the infarct margin and the demarcation zone in OPN-treated animals. Scale bars represent $50 \mu \mathrm{m}$. All graphs show mean and SD; ${ }^{*} p<0.05$; ${ }^{* * *} p<0.001$. 
Arg1 (M2-polarized microglia)

Arg1 (outer core)

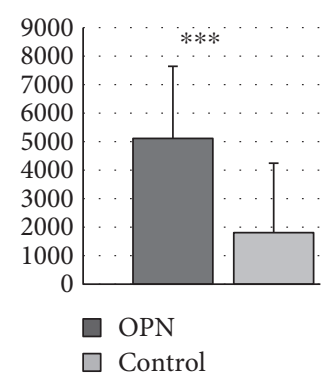

Outer infarct core
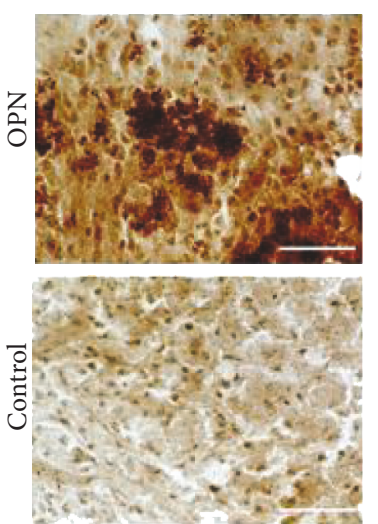

Ym1 (outer core)

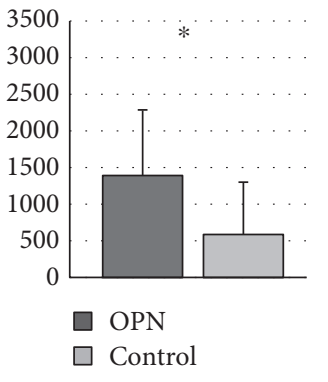

Outer infarct core
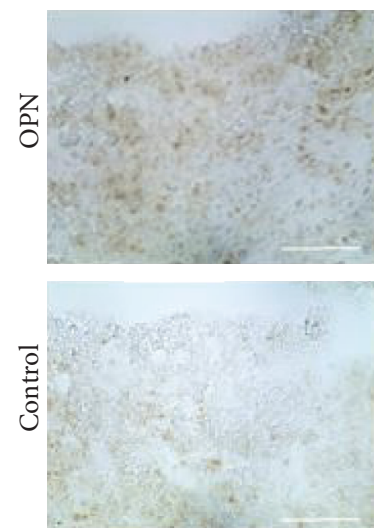

Arg1 (infarct margin)

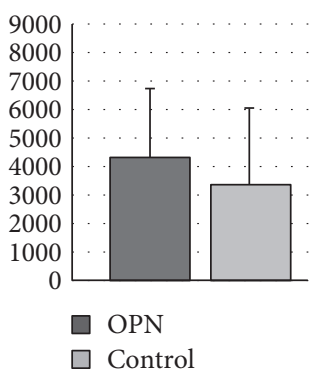

Infarct margin
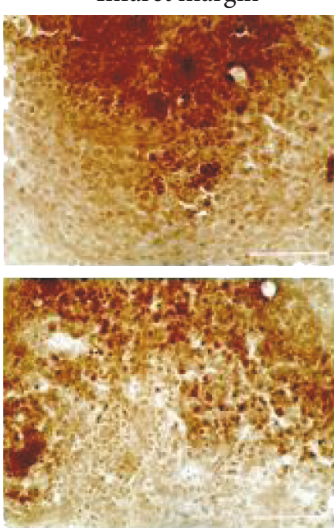

Arg1 (demarcration zone)

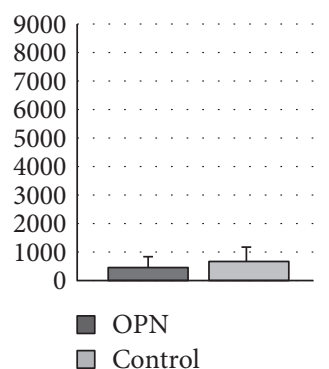

Demarcation zone
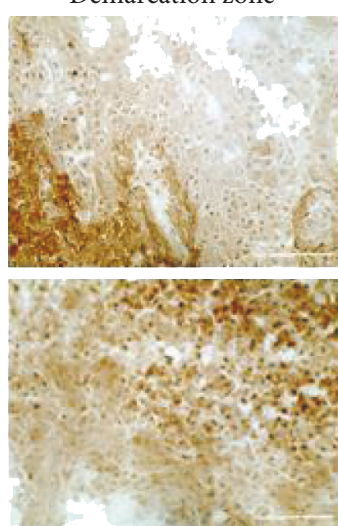

Arg1 (peri-infarct zone)

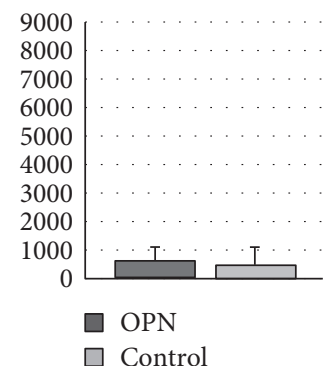

Peri-infarct zone
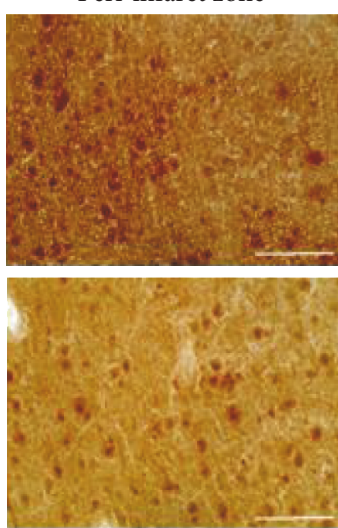

Ym1 (M2-polarized microglia)
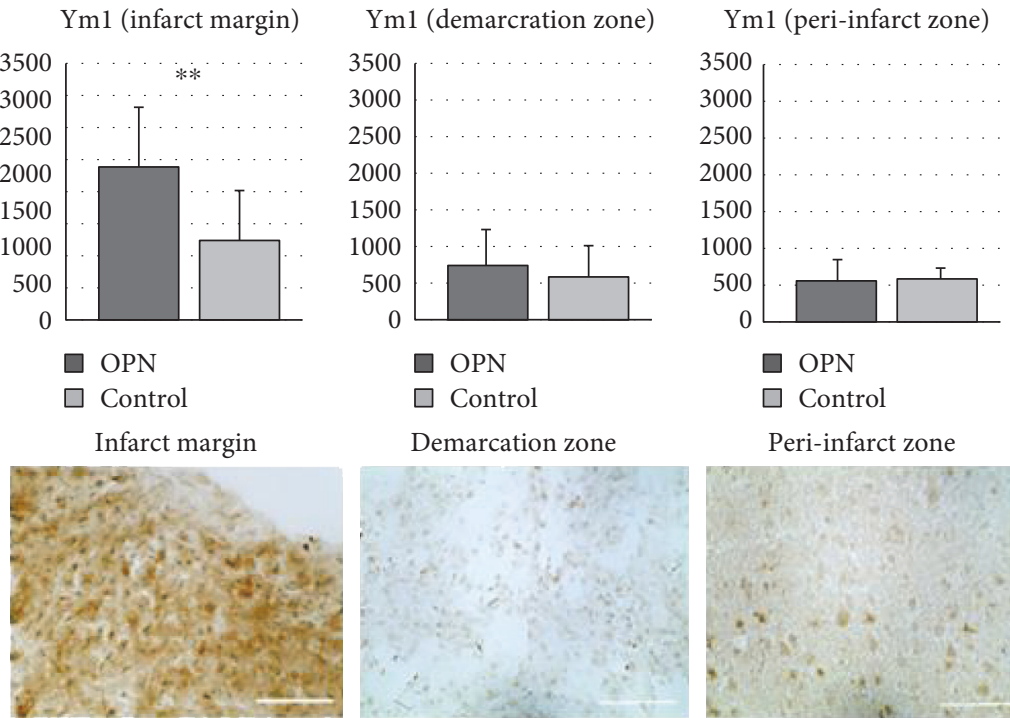

Peri-infarct zone
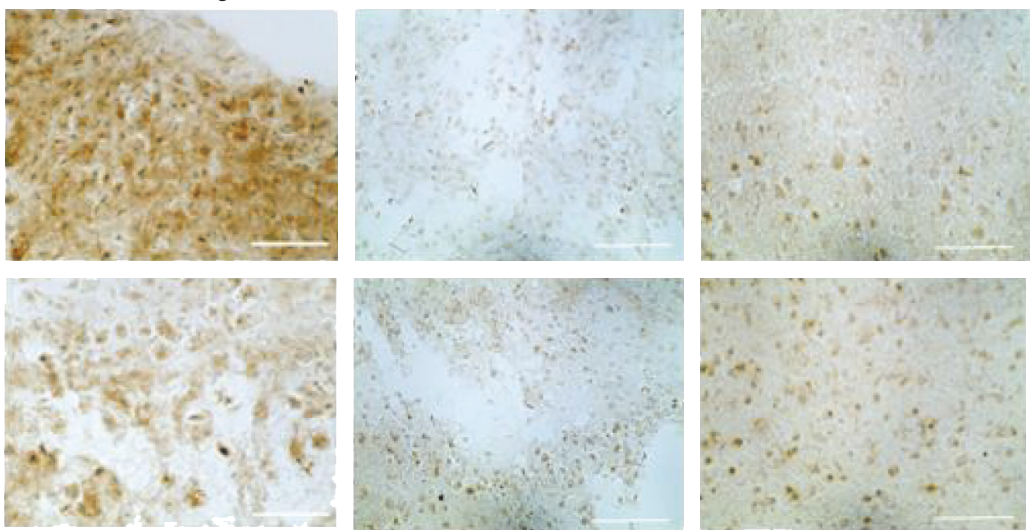

FIGURE 4: OPN modulates the infiltration of microglia subpopulations into distinct infarct subareas (part II: Arg1 and Ym1). M2 subpopulations stained with Arg1 and Ym1 in subareas of photothrombotic infarct after OPN compared to placebo treatment. Arg1+ microglia show an increase in the outer infarct core, while Ym1+ microglia are found enriched in the outer core and infarct margin. Scale bars represent $50 \mu \mathrm{m}$. All graphs show mean and SD; ${ }^{*} p<0.05{ }^{* *} p<0.01{ }^{* * *} p<0.001$. 

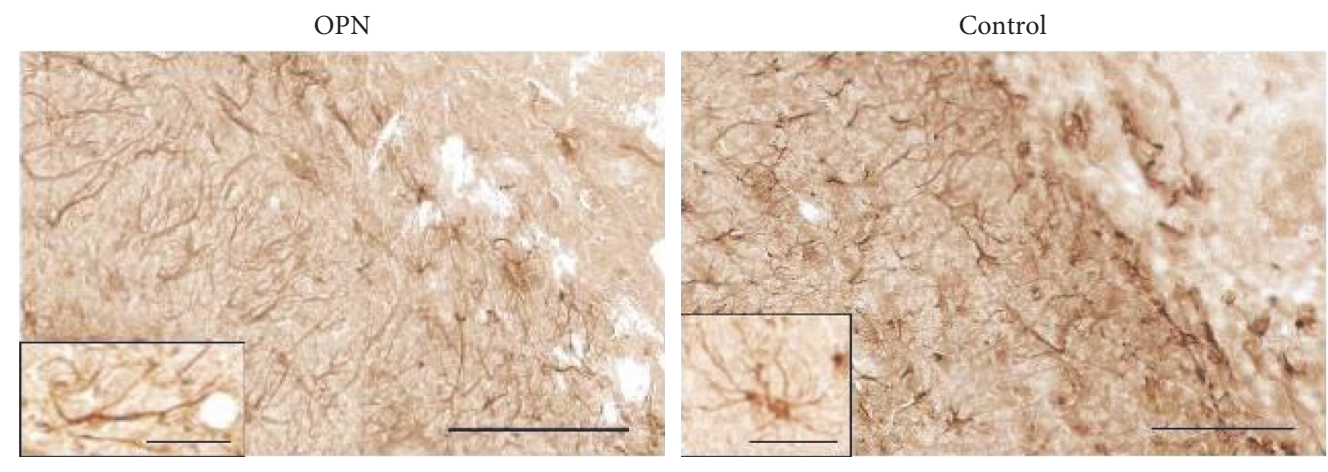

(a)

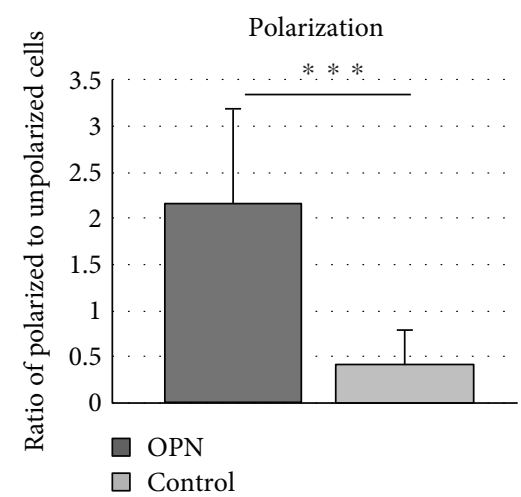

(b)

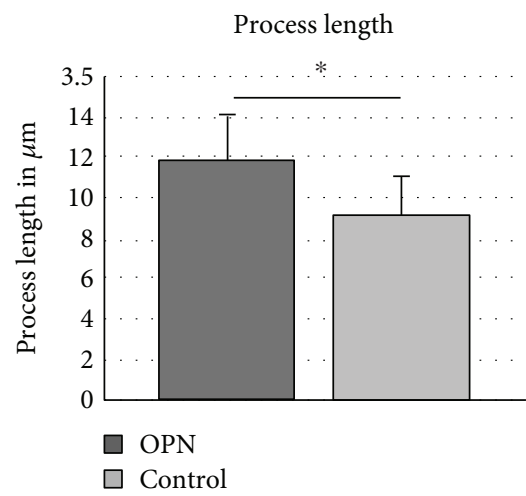

(c)

FIGURE 5: OPN polarizes astrocytes towards the infarct. (a) Infarct margin stained for GFAP showing the majority of astrocytes with processes aligned towards the infarct core in OPN-treated animals, and increased process length in OPN-treated animals. Quantification of changes in polarisation (b) and process length (c). All graphs show mean and SD. ${ }^{*} p<0.05 ;{ }^{* * *} p<0.001$.

lack the ability to switch. While iNOS is the typical marker for M1 microglia, Arginasel signifies the functional antagonist of iNOS, which is the reason why those two enzymes are the favoured markers for M1 and M2 polarization. In the infarct margin, the distinction between M1 and M2 (Arg1, iNOS) was less strict than expected from the single cell level. Many cells appeared to coexpress M1 and M2 markers iNOS and Arg1, respectively, signals indicating a concept of a continuous phenotypical shift rather than distinct microglial subpopulations expressing one or the other $[3,18,29,30]$. We found an inversely proportional correlation between M2 markers (Arg1 $\left[p=0.0322, r^{2}=0.1773\right]$, CD206, $\left.\left[p=0.0022, r^{2}=0.3132\right]\right)$ and infarct size, being compatible with the earlier notion of protective functions of M2 microglia [31, 32]. However, the study design did not allow proving a causative relationship between increased M2 polarization and stroke protection.

Data suggest that better-defined layering of microglia/ macrophage subtypes after stroke is a surrogate for beneficially modifying poststroke inflammation processes. Our results are in line with the assumption that M1/M2 dichotomy is not absolute, as predicted by findings about the Th1/Th2 dichotomy of lymphocyte activation [32]. Rather, $\mathrm{M} 1 / \mathrm{M} 2$ polarization represents a relative dynamic state along a spectrum of activational conditions, and activated microglia may even express both or none of the polarization markers (Figure 1(c)). However, a clear separation of M1 and M2 cells may be beneficial for the outcome after stroke (Figure 2).

Previous studies also described enhanced neovascularisation in the vascular stem cell niche as well as an increased migration of stem cells out of their niche after ischemia $[33,34]$. We have recently reported an increased migration of stem cells after stroke when treated with OPN i.c.v. [35]. Accordingly, OPN might be able to mediate between microglia and stem cells. In line with those previous results, the current study demonstrated an increased vascularization of the infarcted area in OPN-treated animals. Moreover, astrocyte polarization of astrocytes towards the infarct core was increased after OPN treatment, possibly supporting the containment of inflammation. These findings support recent data on lacking impaired astrocyte polarization in OPN-deprived animals [5].

Our data suggest that OPN constitutes a candidate molecule to study the interaction of microglia and endogenous stem cells after stroke and could be a suitable modulating agent in CNS to support repair processes in the CNS.

\section{Conclusion}

Microglial activation and M1 and M2 polarization constitute microglia subpopulations that occur in distinct but overlapping spatial patterns following permanent focal ischemia. Osteopontin drives microglia polarization towards M2 and 
may be involved in locally separating M1 and M2 subpopulations locally. Whether this separation contributes to the beneficial effects of osteopontin warrants further investigation. Future studies should be directed at examining the molecular pathways linking OPN and microglial activation and focus on modifying the $\mathrm{M} 2$ response.

\section{Abbreviations}

Arg1: Arginase 1

CCA: Common carotid artery

DAB: Diaminobenzidine

ECM: Extracellular matrix

GFAP: Glial fibrillary acidic protein

Iba1: Ionizing calcium binding adaptor protein 1

ICA: Internal carotid artery

i.c.v.: Intracerebroventricular

IFN $\gamma$ : Interferon $\gamma$

IgG: Immunoglobulin $\mathrm{G}$

IL: Interleukin

iNOS: Inducible nitric oxide synthase

LPS: Lipopolysaccharide

MCAO: Middle cerebral artery occlusion

MHC: Major histocompatibility complex

NeuN: Neuronal nuclei

NG2: Chondroitin sulfate proteoglycan

NSC: Neural stem cells

NO: $\quad$ Nitric oxide

OPN: Osteopontin

PT: $\quad$ Photothrombosis

ROS: $\quad$ Reactive oxygen species

RNS: Reactive nitrogen species

RT: Room temperature

TGF $\beta$ : Transforming growth factor

TNF $\alpha$ : Tumor necrosis factor $\alpha$

vWF: von Willebrand factor

Ym1: Chitinase 3-like-3.

\section{Ethical Approval}

All applicable international, national, and/or institutional guidelines for the care and use of animals were followed. All animal procedures were in accordance with the German Laws for Animal Protection and were approved by the local animal care committee (Tierschutz-Ausschuss FZ Juelich) as well as local governmental authorities (LANUV NRW, AZ 84-02.04.2011.A169).

\section{Conflicts of Interest}

Jörg Hucklenbroich is an employee of Qiagen GmbH, Hilden, Germany. Michael Schroeter has received a speaker honorarium from Biogen, Miltenyi Biotec, and Grifols. Anne Ladwig, Helene Luise Walter, Antje Willuweit, Karl-Josef Langen, Gereon Rudolph Fink, and Maria Adele Rueger declare that they have no competing interests.

\section{Acknowledgments}

The authors thank Mr. Michael Schoeneck and Ms. Claudia Drapatz for the excellent technical assistance and Dr. med. Rebecca Klein for the reevaluations. This research work was funded by the Köln Fortune Program/Faculty of Medicine, University of Cologne, Germany (106/2012). The authors also thank the Marga and Walter Boll Foundation for generously supporting their lab.

\section{References}

[1] J. R. Weinstein, I. P. Koerner, and T. Möller, "Microglia in ischemic brain injury," Future Neurology, vol. 5, no. 2, pp. 227-246, 2010.

[2] G. Stoll, S. Jander, and M. Schroeter, "Detrimental and beneficial effects of injury-induced inflammation and cytokine expression in the nervous system," Advances in Experimental Medicine and Biology, vol. 513, pp. 87-113, 2002.

[3] R. Orihuela, C. A. McPherson, and G. J. Harry, "Microglial M1/M2 polarization and metabolic states," British Journal of Pharmacology, vol. 173, no. 4, pp. 649-665, 2016.

[4] H. L. Claus, M. Walberer, M. L. Simard et al., "NG2 and NG2positive cells delineate focal cerebral infarct demarcation in rats," Neuropathology, vol. 33, no. 1, pp. 30-38, 2013.

[5] M. Gliem, K. Krammes, L. Liaw, N. van Rooijen, H.-P. Hartung, and S. Jander, "Macrophage-derived osteopontin induces reactive astrocyte polarization and promotes reestablishment of the blood brain barrier after ischemic stroke," Glia, vol. 63, no. 12, pp. 2198-2207, 2015.

[6] M. Rabenstein, S. U. Vay, L. J. Flitsch, G. R. Fink, M. Schroeter, and M. A. Rueger, "Osteopontin directly modulates cytokine expression of primary microglia and increases their survival," Journal of Neuroimmunology, vol. 299, pp. 130-138, 2016.

[7] M. Schroeter, P. Zickler, D. T. Denhardt, H.-P. Hartung, and S. Jander, "Increased thalamic neurodegeneration following ischaemic cortical stroke in osteopontin-deficient mice," Brain: A Journal of Neurology, vol. 129, Part 6, pp. 1426-1437, 2006.

[8] T. Mabuchi, K. Kitagawa, T. Ohtsuki et al., "Contribution of microglia/macrophages to expansion of infarction and response of oligodendrocytes after focal cerebral ischemia in rats," Stroke, vol. 31, no. 7, pp. 1735-1743, 2000.

[9] A.-G. Ceulemans, T. Zgavc, R. Kooijman, S. Hachimi-Idrissi, S. Sarre, and Y. Michotte, "The dual role of the neuroinflammatory response after ischemic stroke: modulatory effects of hypothermia," Journal of Neuroinflammation, vol. 7, no. 1, p. 74, 2010.

[10] I. M. Shah, I. M. Macrae, and M. NapoliDi, "Neuroinflammation and neuroprotective strategies in acute ischaemic stroke from bench to bedside," Current Molecular Medicine, vol. 9, no. 3, pp. 336-354, 2009.

[11] M. Schroeter, M. A. Dennin, M. Walberer et al., "Neuroinflammation extends brain tissue at risk to vital peri-infarct tissue: a double tracer [11C]PK11195- and [18F]FDG-PET study," Journal of Cerebral Blood Flow and Metabolism, vol. 29, no. 6, pp. 1216-1225, 2009.

[12] S. Jander, M. Schroeter, D. D’Urso, C. Gillen, O. W. Witte, and G. Stoll, "Focal ischaemia of the rat brain elicits an unusual inflammatory response: early appearance of CD8+ macrophages/microglia," The European Journal of Neuroscience, vol. 10, no. 2, pp. 680-688, 1998. 
[13] M. Schilling, M. Besselmann, C. Leonhard, M. Mueller, E. B. Ringelstein, and R. Kiefer, "Microglial activation precedes and predominates over macrophage infiltration in transient focal cerebral ischemia: a study in green fluorescent protein transgenic bone marrow chimeric mice," Experimental Neurology, vol. 183, no. 1, pp. 25-33, 2003.

[14] M. Schilling, M. Besselmann, M. Müller, J. K. Strecker, E. B. Ringelstein, and R. Kiefer, "Predominant phagocytic activity of resident microglia over hematogenous macrophages following transient focal cerebral ischemia: an investigation using green fluorescent protein transgenic bone marrow chimeric mice," Experimental Neurology, vol. 196, no. 2, pp. 290-297, 2005.

[15] M. Schroeter, S. Jander, I. Huitinga, O. W. Witte, and G. Stoll, "Phagocytic response in photochemically induced infarction of rat cerebral cortex. The role of resident microglia," Stroke, vol. 28, no. 2, pp. 382-386, 1997.

[16] C. A. Colton and D. M. Wilcock, "Assessing activation states in microglia," CNS \& Neurological Disorders Drug Targets, vol. 9, no. 2, pp. 174-191, 2010.

[17] H. W. Morrison and J. A. Filosa, "A quantitative spatiotemporal analysis of microglia morphology during ischemic stroke and reperfusion," Journal of Neuroinflammation, vol. 10, no. 1, p. 4, 2013.

[18] X. Hu, P. Li, Y. Guo et al., "Microglia/macrophage polarization dynamics reveal novel mechanism of injury expansion after focal cerebral ischemia," Stroke, vol. 43, no. 11, pp. 30633070, 2012.

[19] R. D. Stout, C. Jiang, B. Matta, I. Tietzel, S. K. Watkins, and J. Suttles, "Macrophages sequentially change their functional phenotype in response to changes in microenvironmental influences," The Journal of Immunology, vol. 175, no. 1, pp. 342-349, 2005.

[20] R. Meller, S. L. Stevens, M. Minami et al., "Neuroprotection by osteopontin in stroke," Journal of Cerebral Blood Flow and Metabolism, vol. 25, no. 2, pp. 217-225, 2005.

[21] X. Wang, C. Louden, T. L. Yue et al., "Delayed expression of osteopontin after focal stroke in the rat," Journal of Neuroscience: The Official Journal of the Society for Neuroscience, vol. 18, no. 6, pp. 2075-2083, 1998.

[22] F. Kahles, H. M. Findeisen, and D. Bruemmer, "Osteopontin: a novel regulator at the cross roads of inflammation, obesity and diabetes," Molecular Metabolism, vol. 3, no. 4, pp. 384-393, 2014.

[23] Q. Jin, J. Cheng, Y. Liu et al., "Improvement of functional recovery by chronic metformin treatment is associated with enhanced alternative activation of microglia/macrophages and increased angiogenesis and neurogenesis following experimental stroke," Brain, Behavior, and Immunity, vol. 40, pp. 131-142, 2014.

[24] E. A. Bordt and B. M. Polster, "NADPH oxidase- and mitochondria-derived reactive oxygen species in proinflammatory microglial activation: a bipartisan affair?," Free Radical Biology \& Medicine, vol. 76, pp. 34-46, 2014.

[25] T. L. Sudduth, F. A. Schmitt, P. T. Nelson, and D. M. Wilcock, "Neuroinflammatory phenotype in early Alzheimer's disease," Neurobiology of Aging, vol. 34, no. 4, pp. 1051-1059, 2013.

[26] J. D. Cherry, J. A. Olschowka, and M. K. O’Banion, "Neuroinflammation and M2 microglia: the good, the bad, and the inflamed," Journal of Neuroinflammation, vol. 11, no. 1, p. 98, 2014.
[27] K. S. Rawji and V. W. Yong, "The benefits and detriments of macrophages/microglia in models of multiple sclerosis," Clinical \& Developmental Immunology, vol. 2013, article 948976, 13 pages, 2013.

[28] D. Amantea, G. Micieli, C. Tassorelli et al., "Rational modulation of the innate immune system for neuroprotection in ischemic stroke," Frontiers in Neuroscience, vol. 9, p. 147, 2015.

[29] M. J. Crane, J. M. Daley, O. Houttevan, S. K. Brancato, W. L. Henry, and J. E. Albina, "The monocyte to macrophage transition in the murine sterile wound," PLoS One, vol. 9, no. 1, article e86660, 2014.

[30] L. Arnold, A. Henry, F. Poron et al., "Inflammatory monocytes recruited after skeletal muscle injury switch into antiinflammatory macrophages to support myogenesis," The Journal of Experimental Medicine, vol. 204, no. 5, pp. 1057-1069, 2007.

[31] S. Gordon, "Alternative activation of macrophages," Nature Reviews. Immunology, vol. 3, no. 1, pp. 23-35, 2003.

[32] S. Gordon and F. O. Martinez, "Alternative activation of macrophages: mechanism and functions," Immunity, vol. 32, no. 5, pp. 593-604, 2010.

[33] R. L. Zhang, M. Chopp, C. Roberts et al., "Stroke increases neural stem cells and angiogenesis in the neurogenic niche of the adult mouse," PLoS One, vol. 9, no. 12, article e113972, 2014.

[34] R. Zhang, Z. Zhang, L. Wang et al., "Activated neural stem cells contribute to stroke-induced neurogenesis and neuroblast migration toward the infarct boundary in adult rats," Journal of Cerebral Blood Flow and Metabolism, vol. 24, no. 4, pp. 441-448, 2004.

[35] M. Rabenstein, J. Hucklenbroich, A. Willuweit et al., "Osteopontin mediates survival, proliferation and migration of neural stem cells through the chemokine receptor CXCR4," Stem Cell Research \& Therapy, vol. 6, no. 1, p. 99, 2015. 


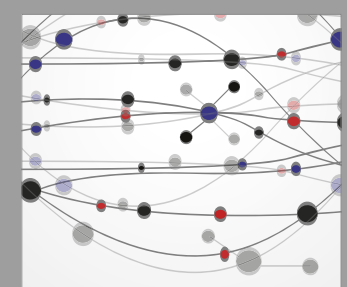

The Scientific World Journal
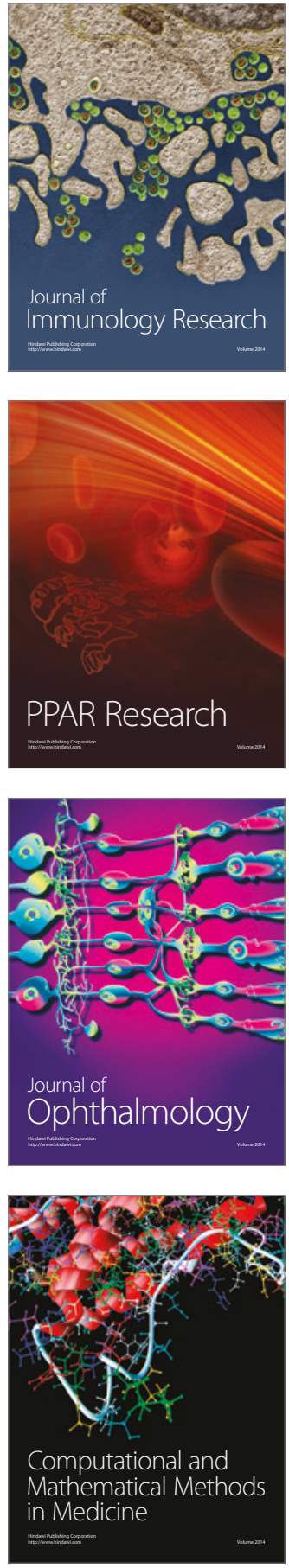

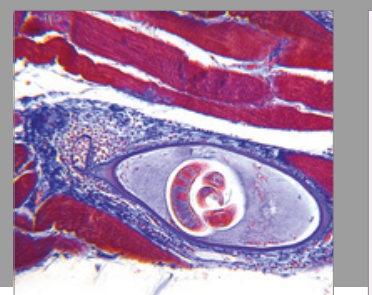

Gastroenterology Research and Practice
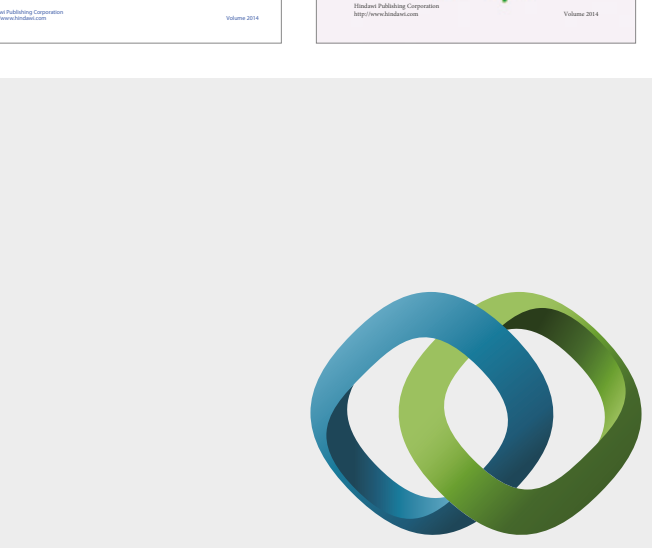

\section{Hindawi}

Submit your manuscripts at

https://www.hindawi.com
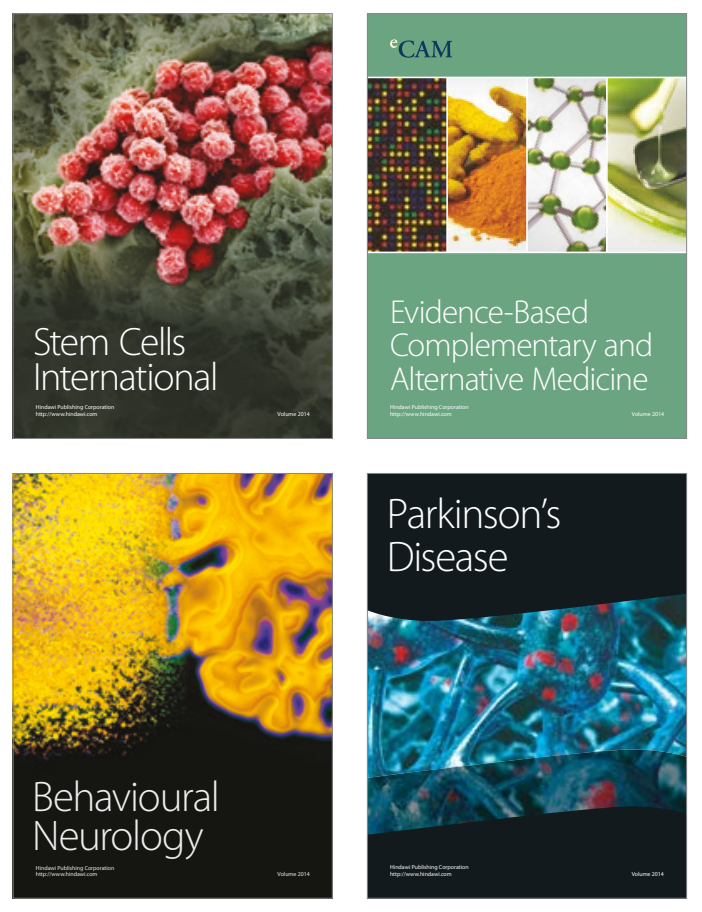
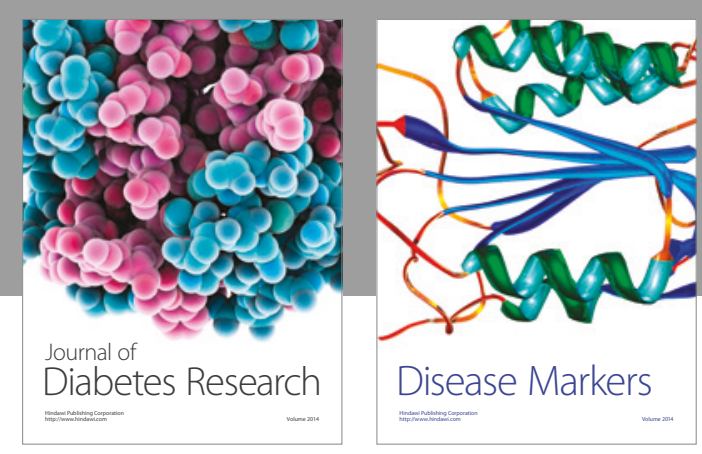

Disease Markers
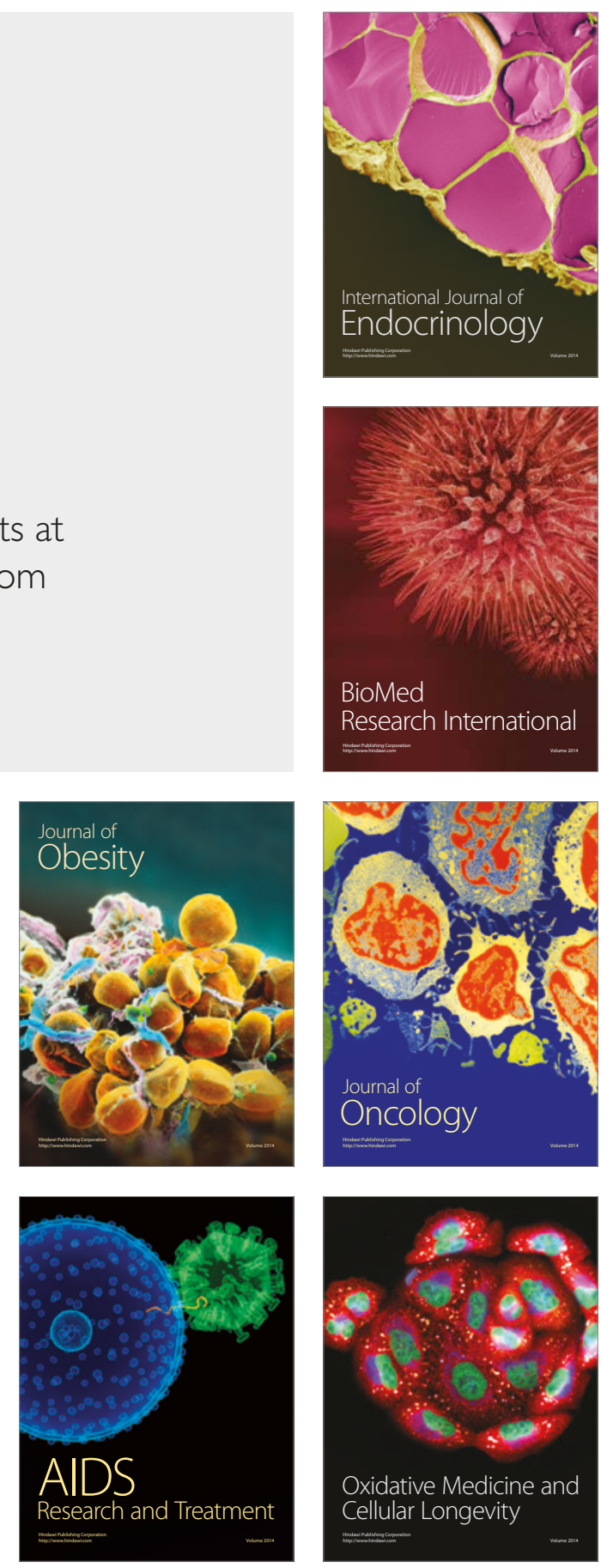